\title{
Announcements
}

\section{Calendar of Forthcoming Meetings}

2002

Apr.

$$
7 \text { - } 11 \text { (Manchester) }
$$

\section{EUROPT(R)ODE
and Biosensors.}

Contact: Dr. R. Narayanaswamy, DIAS, UMIST, P. O. Box 88, Manchester M60 1QD, UK (e-mail: EuroptrodeVI@umist.ac.uk; Website http://www.dias.umist.ac.uk/EuroptrodeVI/).

$21-25$ (Bruges)

$23-26$ (München)

\begin{abstract}
May $25-26$ (Himeji)
June $4-7$ (Granada)
\end{abstract}

Oct. $20-25$ (Kyoto)

Nov. $\quad 17-21$ (Yokohama)
Drug Analysis 2002 (The VIIth International Symposium on Drug Analysis). Contact: Orga-Med Congress Office, Mr. Peter Erard, Project Manager, Essenstraat 77, B-1740 Ternat, Belgium (e-mail: orgamed@ village.uunet.be). ANALYTICA 2002. The 18th International Trade Fair for Instrumental Analysis, Laboratory Technology and Bio Technologies and the ANALYTICA CONFERENCE.

Contact: Messe München GmbH, 81823 München, Germany (e-mail: info@ analytica.de; Website http://www.analytica.de).

The 63rd Symposium on Analytical Chemistry.*

Xth International Symposium on Luminescence Spectrometry—Detection Techniques in Flowing Streams-Quality Assurance and Applied Analysis. Contact: Prof. Dr. Ana Maria Garcia-Campana, Chairwoman, Department of Analytical Chemistry, Faculty of Sciences, University of Granada, Av. Fuentenueva s/n, E-18071 Granada, Spain.

The 18th International Congress of Clinical Chemistry and Laboratory Medicine. Contact: Secretariat of the 18th ICCC 2002, Kyoto, c/o Center for Academic Societies Japan, Osaka, 14th floor, Senri Life Science Centre Bldg., 1-4-2 Shinsenrihigashi-machi, Toyonaka 560-0082, Japan (e-mail: iccckyoto@bcasj.or.jp; Website http://iccc2002.bcasj.or.jp/).

SCOPE/IUPAC International Symposium on Endocrine Active Substances. Contact: Secretariat, K. Kubota, Chemicals Evaluation and Research Institute (CERI), 1-4-25, Kouraku, Bunkyo-ku, Tokyo 112-0004, Japan (e-mail: eds-sympo@cerij.or.jp; Website http://www.cerij.or.jp).

*Contact: The Japan Society for Analytical Chemistry, 26-2, Nishigotanda 1-chome, Shinagawa-ku, Tokyo 141-0031, Japan. 


\section{BUNSEKI KAGAKU \\ Vol. 50 No. 12 December, 2001 \\ Special Articles: Analytical Chemistry for Advanced Technologies}

\section{Accounts}

Miniaturized Analytical System Using Fibrous

\section{Packing Media}

Kiyokatsu Jinno, Yoshihiro Saito and Motohiro Imaizumi 775

Analytical Methodology Supporting Semiconductor

Packaging and Interconnect Technologies

Shin-ichi Wakabayashi 785

\section{Lecture}

Recent Key Issues of Automobiles and Related

Analysis of Materials

Takuya Kondo and Takashi Kuzuya 799

\section{Research Papers}

Rapid Decomposition and Analysis of Refractory Barium Sulfate by Fusion with Ammonium Salt

Ken Matsumoto and Toshihiro Koura 807

Quantitative Determination of $\mathrm{K}^{+}, \mathrm{NH}_{4}^{+}, \mathrm{Na}^{+}, \mathrm{Ca}^{2^{+}}$

and $\mathrm{Mg}^{+}$Cations in Secreted Human Sweat by

Capillary Zone Electrophoresis

Junko Terasawa, Kotaro Mitsuya, Akira Ishii and Takao Tsuda 813

Determination of 3-Dimethylsulfoniopropionate in

Marine Algae by Low-pH Capillary Electrophoresis

Jinghua Zhang, Naoki Nishimura, Mitsuru Abo, Akira Okubo and Sunao Yamazaki 819

Gas-Pressure-Driven Stepwise-Gradient Elution

System for Microcolumn Liquid Chromatography

Toyohide Takeuchi and Lee Wah Lim 825

Analysis of Cadmium and Lead in Sediment by

Isotope-Dilution ICP-MS

Kazumi Inagaki, Akiko Takatsu, Atsuko Nakama, Akira

Uchiumi and Kensaku Okamoto 829

Preparation and Evaluation of Industrial Waste

Incineration Fly Ash Reference Sample

Seiko Iwata, Kazuaki Minamoto, Eiji Fujimori, Koichi Chiba and Hiroki Haraguchi 837

Continuous Monitoring of Nitrogen Oxide

Concentration in Urban Atmosphere and Cross

Correlation with Other Greenhouse Gases

Aritaka Matsunani, Fumie Akimoto, Ippei Kodama, Yuichi

Kamata, Kuniyuki Kitagawa, Norio Arai, Takuya Higuchi,

Akinori Ito and Hiroki Haraguchi 845

\section{Technical Papers}

Rapid Determination of Copper in Steel by

Electrolytic Dissolution/Stripping Voltammetry

Tatsuhiko Tanaka, Keisuke Shitan and Hiroyuki Kondo 855

Shortening the Decomposition Time for Sintered

Alumina by High-Temperature Pressure Acid

Decomposition

Yoshinori Uwamino, Hisashi Morikawa, Akira Tsuge, Kiyoshi Nakane and Toshio Ishizuka 861
Direct Determination of Boron in Iron and Steel by Ion-Pair Reversed-Phase HPLC with a LaboratoryPacked Column

Kazuya Uesawa, Nobuo Uehara, Kiyotaka Ito and Tokuo Shimizu 867

Classificatin of Pressure Sensitive Adhesive Cloth Tapes Based on Data by X-ray Spectroscopic Analysis Using Multivariate Analysis Minemasa Hida, Hiroyasu Satho and Tosiyuki Mitsui 873 Determination of Multi-Elements of Certified Soil Reference Materials for the Analysis of Metal Contents by Instrumental Neutron-Activation Analysis

Yukiko Okada and Shoji Hirai 879

Differential Determination of Traces of

Arsenic(III) and Arsenic(V) in Water by Flow

Injection On-line Preconcentration-Graphite

Furnace AAS

Yoshihiro Hirano, Hiroki Sakurai, Akihiro Endo, Koichi

Oguma and Yasushi Terui 885

Wide-Scale Monitoring Systems for Spilled Oil and

Chemicals in the Ocean Using Macro-Monitoring

and Micro-Detection

Yoshitaka Yamaguchi, Noboru Taguchi, Hiroshi Yamanouchi,

Toshiaki Shibata, Kazuo Hitomi and Susumu Yamagishi 893

Notes

Preparation of an Acetylcholine Sensor Based on an Enzyme-Immobilized Polyion Complex Membrane Soichi Yabuki, Fumio Mizutani and Yoshiki Hirata 899 Study on a Highly Sensitive Determination Method for Protein in Aqueous Solution Using a Quartz Crystal Microbalance

Hiroaki Matsuura, Fumio Mizutani,Yasushi Hasebe and Shunichi Uchiyama 903

Observation of the Rotational Motion of Silver

Aggregation Produced by a Reductive Reaction in a Strong Magnetic Field

Kazuhiro Honda, Hidetoshi Arizono, Nobutaka Yamasaki,

Yoshihito Mori, Shyuko Fujieda, Akira Satoh and Seiichiro

Nakabayashi 907

Water-Content Analysis by Photothermal

Spectrometry with a Near-Infrared Diode Laser

Hirofumi Kawazumi, Takanobu Ukawa and Tomohiro

Watanabe 911

Digest of Doctoral Dissertation Developments of

Simultaneous Analytical Methods of Hazardous

Chemicals and Their Applications to

Environmental Monitoring

Akiko Tanabe 915

Indexes 